\title{
Avaliação da qualidade da visão, na prática da leitura diária, em relação à formatação dos textos
}

\author{
Evaluation of the quality of vision in daily \\ reading habits in relation to text configuration
}

Milton Borrelli ${ }^{1}$, José Ricardo Carvalho Lima Rehder ${ }^{2}$, Isabelle Martins Squarcino ${ }^{3}$, Aleksandra Mendes Teixeira Gonçalves ${ }^{4}$, Flávia Acciarito Piaia ${ }^{5}$, Priscila K. de Mendonça Fernandes ${ }^{5}$, Vânia de Aquino Albres Santiago ${ }^{5}$, Rodrigo Toledo Mota ${ }^{6}$

\section{ResUmo}

Objetivo: Este estudo avaliou o comportamento da população em relação à capacidade de leitura considerando o tamanho das fontes dos textos, com o objetivo de identificar padrões mínimos acessíveis de tamanhos de letras. Métodos: O método escolhido utilizou de um pesquisa aplicada nos anos de 2007 e 2008, quando foram entrevistados 400 pacientes e acompanhantes do Hospital Estadual Mário Covas de Santo André, com idade a partir dos 40 anos. Todos foram submetidos a um teste de leitura de texto com 5 tamanhos diferentes de fonte. Resultados: A maioria dos entrevistados $(79,75 \%)$ relatou dificuldades de leitura, optando pelo maior tamanho de letra dentre os padrões apresentados, concluindo-se que o tamanho da letra tem grande influência na qualidade da leitura. Conclusão: Este achado justifica alertar a sociedade sobre as desordens visuais que acometem as pessoas por volta da faixa etária de 40 anos e indica o tamanho de fonte 14 como ideal para melhorar a leitura da população com presbiopia ou outras desordens refrativas.

Descritores: Presbiopia; Leitura; Acesso à informação; Qualidade de vida

\footnotetext{
${ }^{1}$ Livre-docente, Professor de Comunicação e Expressão da Faculdade de Medicina do ABC - FMABC - Santo André (SP), Brasil; ${ }^{2}$ Professor Titular da Disciplina de Oftalmologia da Faculdade de Medicina do ABC - FMABC - Santo André (SP), Brasil;

${ }^{3}$ Coordenador do Curso de Fisioterapia da Faculdade de Medicina do ABC - FMABC - Santo André (SP), Brasil e do Centro de Reabilitação do Hospital Estadual Mário Covas - Santo André (SP), Brasil;

${ }^{4}$ Fisioterapeuta do Centro de Reabilitação do Hospital Estadual Mário Covas - Santo André (SP), Brasil;

${ }^{5}$ Intérprete de LIBRAS - Acessibilidade e comunicação do Hospital Estadual Mário Covas - Santo André (SP), Brasil;

${ }^{6}$ Acadêmico do Curso de Medicina Faculdade de Medicina do ABC - FMABC - Santo André (SP), Brasil.
} 


\section{$\underline{\text { INTRODUÇÃO }}$}

A leitura é fundamental para a aquisição de conhecimento e consequentemente para o desenvolvimento intelectual, fatores essenciais na promoção da interação social. Os problemas visuais, advindos do envelhecimento, não assistidos por falta de acesso a tratamento oftalmológico, podem prejudicar a eficiência da leitura. As desordens visuais constituem graves problemas de saúde para os idosos, com impacto significativo nas atividades diárias e, portanto na qualidade de vida, como por exemplo, dificuldade para dirigir e quedas associadas a fraturas ${ }^{(1)}$.

A degeneração macular relacionada à idade é um dos principais fatores de cegueira em países industrializados ${ }^{(1)}$, podendo desencadear o relato de "analfabetismo adquirido" pela população acometida pelo desenvolvimento da doença ${ }^{(2)}$.

Outras causas de prejuízo da visão relacionadas à idade são: presbiopia, catarata, glaucoma e retinopatia diabética ${ }^{(1)}$.A presbiopia é a perda do poder de acomodação da visão que ocorre no envelhecimento ${ }^{(3)}$, podendo estar associada a outras desordens refrativas como a hipermetropia, a miopia e também ao astigmatismo $^{(4)}$.

A dificuldade para leitura pode estar relacionada a fatores econômicos ou descuido pessoal por falta de acesso ao tratamento e, é agravada por publicações que utilizam caracteres de textos cada vez menores sem considerar se o olho humano tem capacidade de acompanhar o ritmo da revolução tecnológica editorial. Motivados para minimizar tal dificuldade causada por letras demasiadamente pequenas, nos Estados Unidos foi criado um novo ramo editorial com obras impressas nas chamadas "letras gigantes" e, no Brasil, existe também as publicações com a denominação de "Letrão" (5-6). A única regulamentação existente refere-se a Lei Federal no 11.785 , que prevê a padronização de tamanho de letra apenas para contratos de prestação de serviços e de compra e venda, estabelecendo o tamanho de letra 12 como padrão mínimo ${ }^{(7)}$. Estudos sobre alterações fisiológicas na fase senil ou présenil que identificam perda da habilidade de detectar detalhes finos advindas da idade (redução na quantidade de luz que alcança a retina em idosos $)^{(8)}$ corroboram para com os objetivos deste trabalho.

Este estudo mensurou a capacidade de leitura em relação ao tamanho de letra em textos usuais com o propósito de identificar padrões mínimos acessíveis de tamanhos de letras. A partir disso, ampliar a possibilidade da leitura em situações dos textos em bulas de remédio, manuais de equipamentos, catálogos telefônicos, cardápios de restaurantes, livros, revistas ou qualquer publicação escrita presente na vida diária da população.

\section{Métodos}

Através de consulta a várias editoras de diferentes segmentos que foram contatadas a fim de diagnosticar o padrão atual de tamanho das fontes das publicações do país, constatou-se ser usual a fonte 12 como padrão, com variações entre a mínima fonte 10 e a máxima fonte 18.

Foram entrevistadas 400 pessoas com idade superior a 40 anos, sendo 274 mulheres e 126 homens, no ambulatório de especialidades médicas do Hospital Estadual Mário Covas, em 2007 e 2008, após lerem e assinarem o termo de consentimento livre e esclarecido.

Esta população, voluntária, foi dividida em 4 grupos de 100 pessoas, separadas independentemente do sexo e de acordo com as seguintes faixas etárias: Grupo 1 (40 a 45 anos); Grupo 2 (46 a 50 anos); Grupo 3 (51 a 55 anos) e Grupo 4 (56 anos ou mais).

Todos responderam a um questionário sendo que,na última questão, foi utilizado um texto ${ }^{(9)}$ impresso em 5 tamanhos diferentes (Quadro 1), todos utilizando a fonte Courier $\mathrm{New}$, que oferece melhor leitura para aqueles com problemas de visão $\mathrm{o}^{(10-11)}$. Os textos foram identificados por letras, sendo: texto A (fonte tamanho 10), texto B (tamanho 11), texto C (tamanho12), texto D (tamanho 14), e o texto E (com fonte de tamanho 15). A leitura foi feita sob iluminação ambiente ou natural, respeitando a distância padrão de $33 \mathrm{~cm}$ e com os recursos visuais próprios ou ortéticos dos voluntários.

Os textos de A a E foram apresentados aos entrevistados, que tinham a tarefa de escolher entre eles, qual não oferecia dificuldade de leitura para cada uma das 3 situações do quadro a seguir (Quadro 2).

\section{Resultados}

A dificuldade para leitura foi relatada por $79,75 \%$ dos voluntários (gráfico 1), sendo mais incidente no grupo 3 , de faixa etária entre 51 e 55 anos, mas também muito presente a partir dos 45 anos - a partir do grupo 2 (tabela 1 ).

O "tamanho da letra" foi apontado como a principal causa da dificuldade para leitura, em todos os grupos, de 1 a 4 , com 47, 49, 43 e $42 \%$ dos entrevistados respectivamente, totalizando $45,25 \%$ destes; este motivo esteve associado a problemas como acesso e desinteresse à leitura, somando-se $75,75 \%$ dos entrevistados; problemas com o acesso à leitura foram indicados como principal causa por $14,75 \%$ dos voluntários e, como causa associada aos mesmos fatores já citados, por $36 \%$ da população do estudo; apenas 9,5\% relatou desinteresse pela leitura como causa principal e $25,25 \%$ como fator associado ao desestímulo à mesma (tabela 2).

A maioria dos voluntários, $69 \%$, afirmou utilizar correção da visão com óculos ou lentes de contato (tabela 3). Destes, $40,75 \%$ faziam correção para longe e per- 


\section{Quadro 1}

Modelo dos textos impressos com as fontes de tamanhos de letras 10,11, 12, 14 e 15, utilizadas no estudo

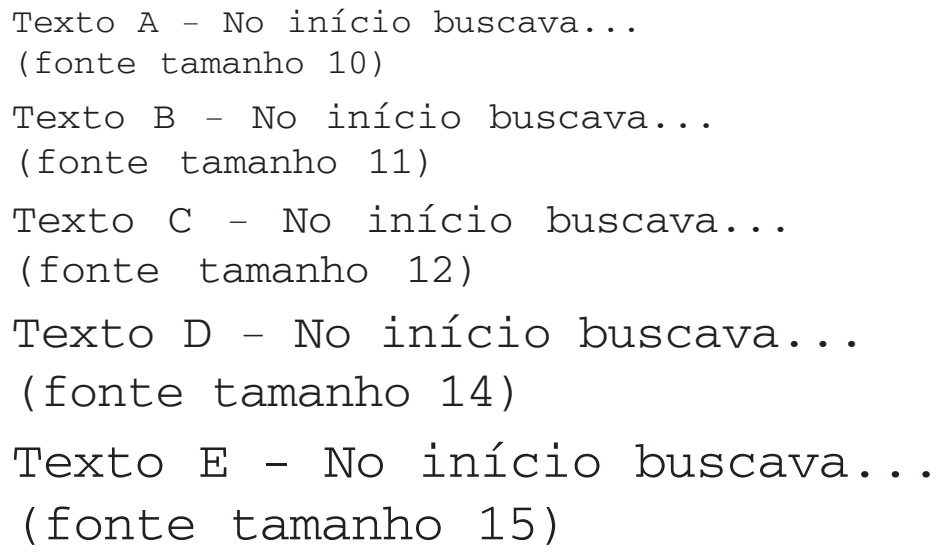

to, $28,75 \%$ somente para perto e $7,5 \%$ para longe (tabela 4). Relataram que não possuem prescrição de correção, $23 \%$ da população do estudo.

Voluntários que já realizaram ao menos uma consulta com oftalmologista somam 79,75\% (tabela 5) e, destes, 59,5\% realizam-no com frequência igual ou menor a um ano (tabela 6) sendo a adesão maior na faixa etária acima de 56 anos (gráfico 2).

Não realizam acompanhamento, $16,5 \%$ da população estudada, sendo que a maioria, $33 \%$ desta situa-se em faixa etária menor (40 a 45 anos) (tabela 7 e gráfico 3).

Na questão 7 (leitura de um mesmo texto com diferentes tamanhos de fonte), na qual os voluntários deviam escolher o tamanho de fonte ideal para leitura de livros (GL), jornais (GI), revistas e cardápios de restaurantes (GR), mais de $70 \%$, em média, escolheu os tamanhos de fonte 14 e 15 sendo que a última, fonte 15 , foi escolhida por $40,75 \%$ dos entrevistados para os três tipos de material de leitura (tabela 8 e gráfico 4 ).

\section{Dıscussão}

Apesar da dificuldade para a leitura atingir todas as faixas etárias, o segmento mais prejudicado, $24,5 \%$ da população brasileira ${ }^{(12)}$, é o dos indivíduos afetados pela presbiopia, desordem refrativa mais comum na vida adulta e relacionada à diminuição da amplitude da acomodação ${ }^{(13)}$, que se manifesta em torno dos 40 anos em indivíduos emétropes e hipermétropes exigindo o uso de óculos para leitura ou lentes de contato ${ }^{(14)}$.

O presente estudo corrobora com as informações acima, apontando que a dificuldade para a leitura está presente na vida da maioria dos entrevistados, $79,75 \%$, com predominância no grupo etário a partir dos 46 anos

\section{Quadro 2}

\section{Situações de leitura}

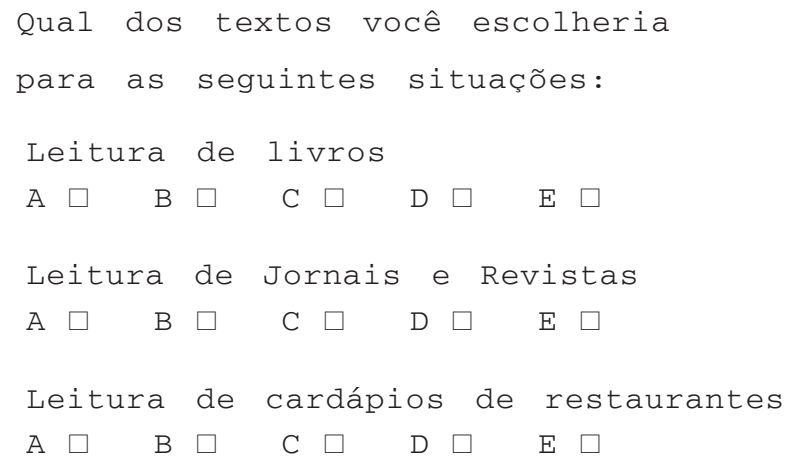

de idade, sendo que $69,5 \%$ utilizam lentes de correção para a visão. Destes, $40,75 \%$ utilizam correção para longe e perto, $28,75 \%$ somente para perto e $7,5 \%$ somente para longe. Com esses dados, acrescenta-se que, apesar da limitação apresentada, 10,25\% dos estudados não a compensam com lentes corretivas, o que poderia estar relacionado com o alto índice de pessoas que nunca se consultaram com oftalmologista $(20,25 \%)$ ou que não o fazem a mais de um ano (40,5\%).

Os resultados mostram também, diferença entre o número de pessoas que possuem prescrição de lentes de correção (77\%) ao daquelas que a utilizam (69\%), denotando um percentual de $08 \%$ que dos entrevistados que possuem receita, portanto deveriam usar óculos ou lente, mas não o fazem.

Os voluntários que realizam acompanhamento com oftalmologista com frequência igual ou menor a 

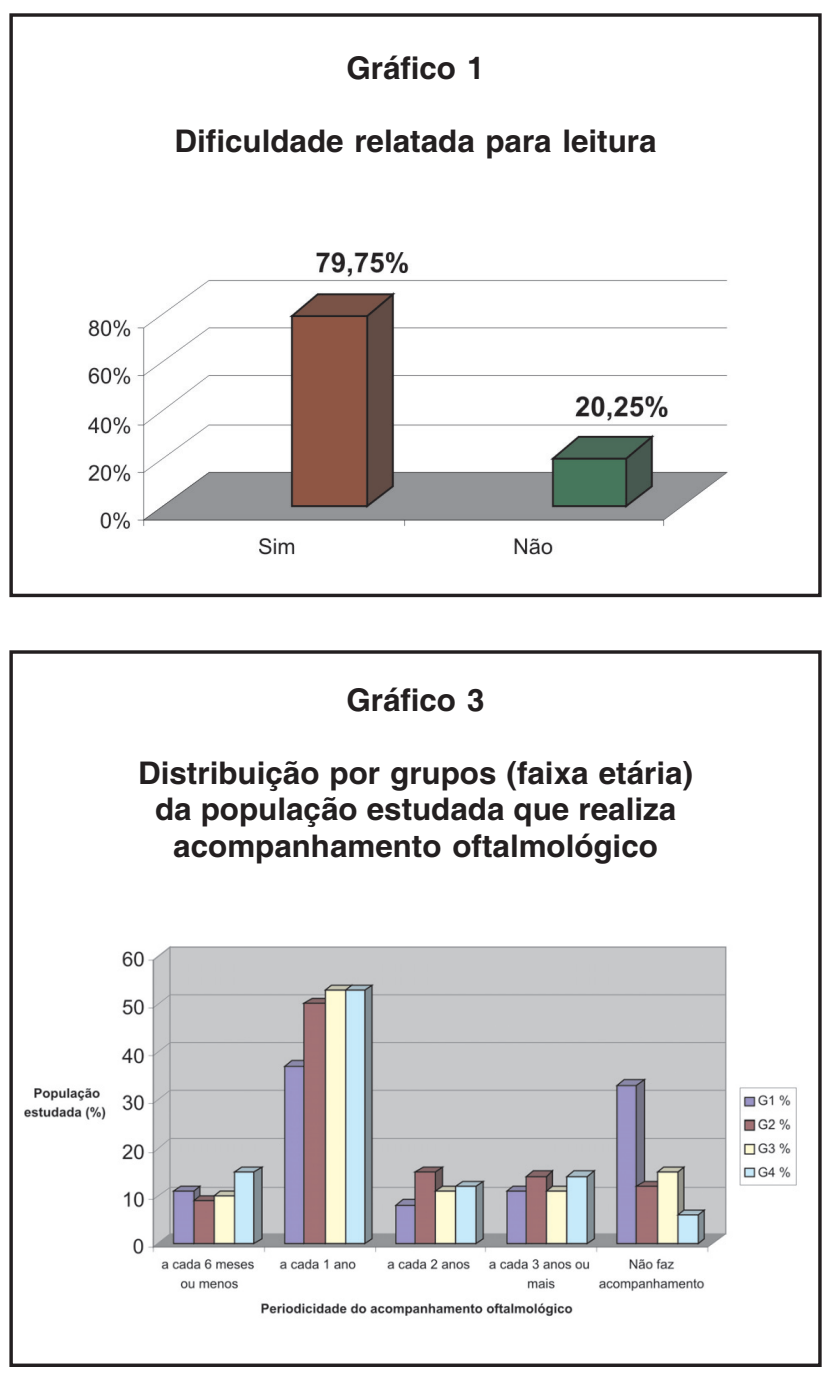

um ano somam 59,5\%; não realizam acompanhamento $16,5 \%$ da população estudada, sendo que $33 \%$ desta situa-se na faixa etária menor ( 40 a 45 anos). A perturbação da acuidade visual, um dos sinais de senilidade, identifica a necessidade de acompanhamento oftalmológico regular, sendo recomendado que, a partir dos 40 anos, seja feito acompanhamento anual com oftalmologista ${ }^{(15)}$.

Relacionando as questões de dificuldade de leitura e o tamanho de letra escolhidos na última questão, pudemos observar que a maioria das pessoas acima de 40 anos (mais de 70\%) refere que o tamanho da letra usualmente utilizado em cardápios, revistas e livros é o fator mais prejudicial ao acesso à informação escrita e que os tamanhos ideais de fonte seriam os maiores, $14 \mathrm{e}$ 15. Este fato pode ser devido ao conforto visual promovido pelo caractere maior. O tamanho dos caracteres é citado como prioridade na maior parte das bibliografias em relação à legibilidade dos textos, devendo ser considerado também o tipo de letra, contraste desta com o
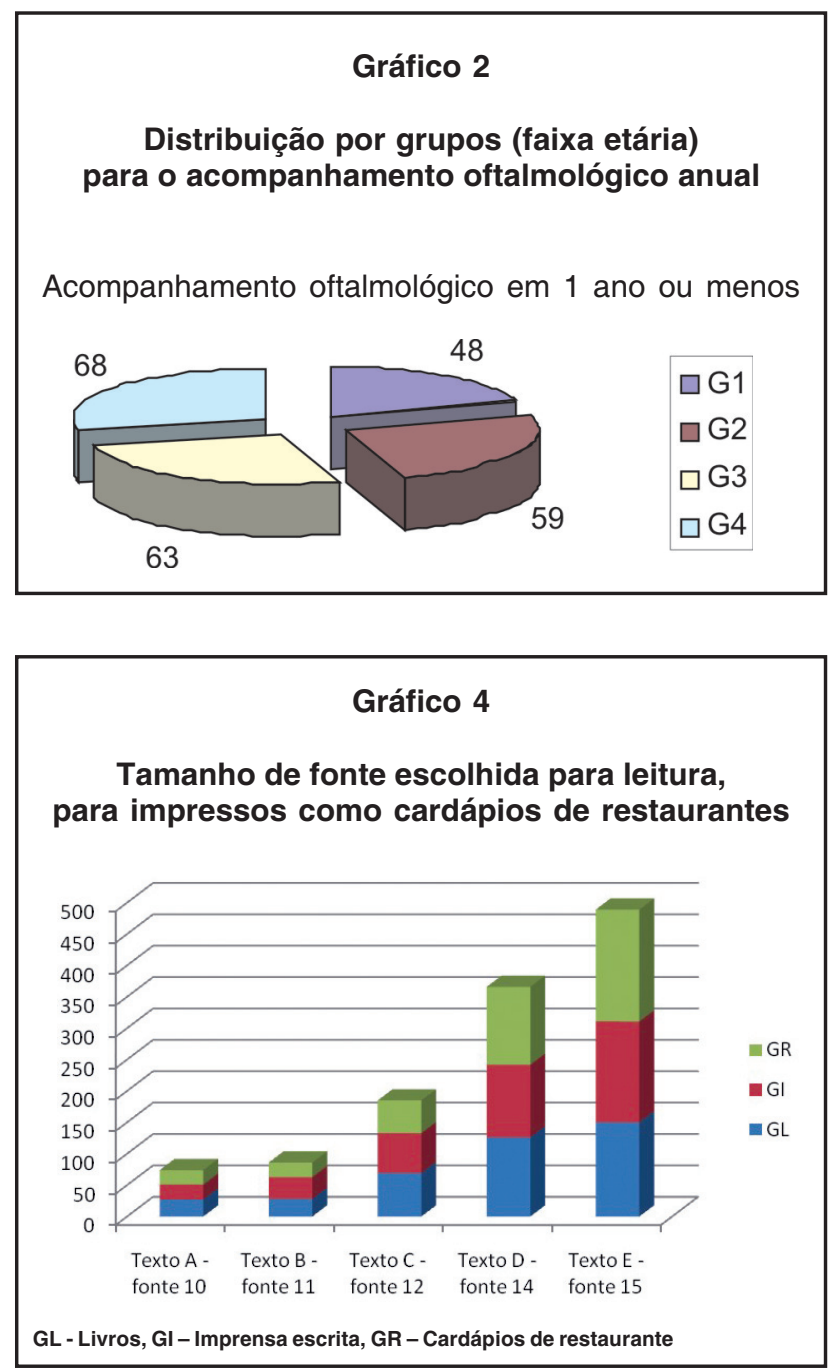

fundo, espaçamentos e linguagem ${ }^{(16)}$.

No Brasil, o artigo 54 do Código do de Defesa do Consumidor ${ }^{(7)}$, estipula que o texto de contratos seja redigido no mínimo com tamanho de letra 12, essa medida fortalece as indagações deste trabalho, pois sendo necessário a padronização de tamanho de letra para a leitura de um contrato visando ao aumento da legibilidade dos textos, entende-se que o mesmo deva ser feito para leituras cotidianas.

Outro exemplo que demonstra a importância do tema é a tecnologia utilizada em algumas páginas da internet através do ícone que permite ao usuário aumentar o tamanho da letra da página de acordo com sua necessidade (Figura 1).

$\mathrm{O}$ aumento do tamanho da fonte significa maior acessibilidade aos conteúdos escritos. A leitura confortável proporciona às pessoas a capacidade de participar de maneira mais completa às discussões da sociedade, ou até mesmo executar atividade simples como procurar o ende- 
Tabela 1

Dificuldade para leitura referida

\begin{tabular}{lccccccc}
\hline & \multicolumn{7}{c}{ Grupos } \\
Dificuldade para leitura & G1 \% & G2 \% & G3 \% & G4 \% & Total & \% \\
\hline Sim & 58 & 86 & 91 & 84 & 319 & 79,75 \\
Não & 42 & 14 & 9 & 16 & 81 & 20,25 \\
Somatório & 100 & 100 & 100 & 100 & 400 & 100 \\
\hline
\end{tabular}

Tabela 2

Motivos da dificuldade para leitura

\begin{tabular}{lcccccc}
\hline $\begin{array}{l}\text { Motivos da dificuldade } \\
\text { para leitura }\end{array}$ & $\mathbf{G 1} \%$ & $\mathbf{G 2} \%$ & $\mathbf{G 3} \%$ & $\mathbf{G 4} \%$ & Total & \% \\
\hline 1 - Tamanho da letra & 47 & 49 & 43 & 42 & 181 & 45,25 \\
2 - Acesso à leitura & 13 & 10 & 29 & 7 & 59 & 14,75 \\
3 - Falta de interesse & 20 & 5 & 5 & 8 & 38 & 9,5 \\
4 - Itens 1 e 2 & 6 & 19 & 6 & 28 & 59 & 14,75 \\
5 - Itens 1 e 3 & 8 & 11 & 12 & 6 & 37 & 9,25 \\
6 - Itens 1, 2 e 3 & 6 & 6 & 5 & 9 & 26 & 6,5 \\
Somatório & 100 & 100 & 100 & 100 & 400 & 100 \\
Tamanho da letra & 67 & 85 & 66 & 85 & 303 & $75,75 \%$ \\
Acesso à leitura & 25 & 35 & 40 & 44 & 144 & $36 \%$ \\
Falta de interesse & 34 & 22 & 22 & 23 & 101 & $25,25 \%$ \\
\hline
\end{tabular}

Tabela 3

Utilização de óculos para leitura

\begin{tabular}{lcccccc}
\hline \multicolumn{7}{c}{ Grupos } \\
Usa óculos para ler & G1 \% & $\mathbf{G 2} \%$ & $\mathbf{G 3} \%$ & $\mathbf{G 4} \%$ & Total & \% \\
\hline Sim & 47 & 69 & 79 & 81 & 276 & 69 \\
Não & 53 & 31 & 21 & 19 & 124 & 31 \\
\hline
\end{tabular}

Tabela 4

Utilização de óculos para longe ou perto

\begin{tabular}{|c|c|c|c|c|c|c|}
\hline \multirow[b]{2}{*}{$\begin{array}{l}\text { Usa óculos para longe } \\
\text { ou para perto }\end{array}$} & \multirow[b]{2}{*}{ G1 \% } & \multicolumn{2}{|c|}{ Grupos } & \multirow[b]{2}{*}{ G4 \% } & \multirow[b]{2}{*}{$\begin{array}{c}\text { Total } \\
\text { parcial }\end{array}$} & \multirow[b]{2}{*}{$\%$} \\
\hline & & G2 \% & G3 \% & & & \\
\hline Longe & 9 & 8 & 4 & 9 & 30 & 7,5 \\
\hline Perto & 18 & 35 & 31 & 31 & 115 & 28,75 \\
\hline Longe e perto & 16 & 38 & 55 & 54 & 163 & 40,75 \\
\hline Não tem prescrição & 57 & 19 & 10 & 6 & 92 & 23 \\
\hline Somatório & 100 & 100 & 100 & 100 & 400 & 100 \\
\hline
\end{tabular}


Tabela 5

Consulta realizada com oftalmologista

\begin{tabular}{lcccccc}
\hline & \multicolumn{7}{c}{ Grupos } \\
$\begin{array}{l}\text { Já realizou consulta } \\
\text { com oftalmologista }\end{array}$ & G1 \% & G2 \% & G3 \% & G4 \% & Total & \% \\
\hline Sim & 58 & 85 & 83 & 93 & 319 & 79,75 \\
Não & 42 & 15 & 17 & 7 & 81 & 20,25 \\
\hline
\end{tabular}

Tabela 6

Acompanhamento anual com oftalmologista por faixa etária

\begin{tabular}{lcccccc}
\hline \multicolumn{7}{c}{ Grupos } \\
Frequência da consulta & G1 \% & G2 $\%$ & G3 \% & G4 \% & Total & \% \\
\hline A cada 6 meses ou - & 11 & 9 & 10 & 15 & 45 & 11,25 \\
A cada 1 ano & 37 & 50 & 53 & 53 & 193 & 48,25 \\
Total & 48 & 59 & 63 & 68 & 238 & 59,5 \\
\hline
\end{tabular}

Tabela 7

Frequência do acompanhamento oftalmológico

\begin{tabular}{lcccccc}
\hline \multicolumn{7}{c}{ Grupos } \\
Frequiência da consulta & G1 \% & G2 \% & G3 \% & G4 \% & Total & \% \\
\hline a cada 6 meses ou - & 11 & 9 & 10 & 15 & 45 & 11,25 \\
a cada 1 ano & 37 & 50 & 53 & 53 & 193 & 48,25 \\
a cada 2 anos & 8 & 15 & 11 & 12 & 46 & 11,5 \\
a cada 3 anos ou + & 11 & 14 & 11 & 14 & 50 & 12,5 \\
Não faz acompanhamento33 & 12 & 15 & 6 & 66 & 16,5 \\
\hline
\end{tabular}

Tabela 8

Escolha do texto conforme os tamanhos de letras 10, 11, 12, 14 e 15 para livros, jornais, revistas e cardápio

\begin{tabular}{lccccc}
\hline Tamanhos de fonte & GL & GI & GR & Média & \% \\
\hline Texto A - fonte 10 & 27 & 24 & 23 & 24,67 & 6,17 \\
Texto B - fonte 11 & 28 & 35 & 23 & 28,67 & 7,17 \\
Texto C - fonte 12 & 69 & 64 & 52 & 61,67 & 15,42 \\
Texto D - fonte 14 & 126 & 116 & 124 & 122,00 & 30,50 \\
Texto E - fonte 15 & 150 & 161 & 178 & 163,00 & 40,75 \\
Somatório & 400 & 400 & 400 & 400 & 100,00 \\
\hline
\end{tabular}

GL - Livros, GI - Imprensa escrita, GR - Cardápios de restaurantes 


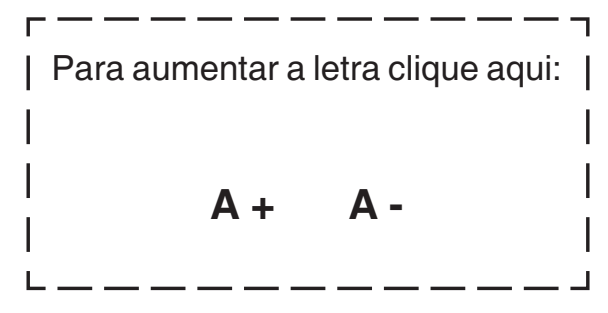

Figura 1 - Exemplo de ícone para aumento de letra na Internet

reço em uma lista telefônica ou uma palavra no dicionário.

A acessibilidade significa, entre outros conceitos, a inexistência de barreiras. O tamanho de letra, tão fácil de ser alterado não pode continuar sendo uma "barreira", tendo em vista a constante queda na taxa de natalidade e aumento da expectativa de vida no Brasil, que caracterizam o envelhecimento da população brasileira ${ }^{(17)}$.

\section{CONCLUSÃO}

Os resultados da pesquisa confirmam a existência de dificuldade para acesso às informações impressas a partir de 40 anos de idade, impedindo a realização de leitura confortável ou fluente como atividade de lazer. Diante do exposto é inegável que a ampliação dos níveis de legibilidade dos textos é essencial. Constitui-se então a proposta deste artigo de padronizar tamanho mínimo dos caracteres em fonte 14 para todos os textos longos, abrangendo a imprensa escrita, a literatura, informativos e também da mídia digital, sendo que a última deve usar o recurso tecnológico de aumento de letra citado no texto. Para cardápios de restaurante, folders e outros impressos promocionais indica-se também o padrão mínimo fonte 14.

\section{AbStRACT}

Objective:This study evaluated the behavior of a population in relation to reading capacity considering font sizes of the texts with the objective of identifying minimum patterns accessible of font sizes. Methods: Four hundred participants and their companions over the age of forty were interviewed at the state hospital Mário Covas of Santo André between 2007 and 2008. They were given a reading test with five different font sizes. Results: The majority (79.75\%) reported having difficulty with reading. Participants preferred larger font size among the samples that were given, concluding that reading is largely influenced by font size. Conclusion: This discovery justifies alerting society about disorders that occur with people over the age of forty, indicating the number 14 as the ideal font size to improve reading in people who suffer from presbyopia and other refractive disorders.

Keywords: Presbyopia; Reading; Access of information; Quality of life

\section{REFERÊNCIAS}

1. Arroyo JG. Age-related macular degeneration: epidemology, etiology, and diagnosis. [Internet - UpToDate, 2008]. [citado 2008 Dez 22]. Disponível em URL: http://www.uptodate.com.

2. Watson GR, Wright V, De L'Aune W. The efficacy of comprehension training and reading practice for print readers with macular loss. J Vis Impair Blind. 1992;86(1):37-43.

3. Vaughan D, Asbury T, Riordan-Eva P. Oftalmologia geral. 15a ed. São Paulo: Atheneu; 2003. p. 365.

4. Guyton AC, Hall JE. Tratado de fisiologia médica. 11a ed. Rio de Janeiro: Elsevier; 2006. Unidade X, Capítulo 49 O Olho I: Óptica da visão. p. 619.

5. Bíblia Sagrada: Letra gigante [Internet]. [citado 2009 Mar 9]. Disponível em: www.livrariasaraiva.com.br/produto/ produto.dll/detalhe? pro_id=1995129\&ID= C8F544827D903030E08391148.

6. Revista coquetel: Linha grande e gigante [Internet]. [citado 2009 Mar 3]. Disponível em: http://www.coquetel.com.br/ templates/coquetel/publicaçao/publicaçao_list.osp? CodCanal $=05 \&$ CodCatalogo $=13729 \&$ visualiza $=$ true .

7. Brasil. Presidência da República. Casa Civil. Subchefia para Assuntos Jurídicos. Lei №. 11.785, de 22 de setembro de 2008. Altera o parágrafo $3^{\circ}$ do art. 54 da Lei ${ }^{\circ} 8.078$, de 11 de setembro de 1990 - Código de Defesa do Consumidor - CDC, para definir tamanho mínimo da fonte em contratos de adesão. [Internet]. [citado 2008 Nov 25]. Disponível em: http://www.planalto.gov.br/ ccivil_03/_ato2007-2010/2008/lei/111785.htm

8. D'Ottaviano EJ. Sistema nervoso e $3^{\text {a }}$ idade. $2^{\text {a }}$ Parte. Argumento. Rev Fac Educ Ciênc Letras Psicol Padre Anchieta. 2001;3(5):29-46.

9. Borrelli M. Histórias. São Paulo: Scortecci; 2007. "O filósofo da praça". p.100.

10. Mansfield JS, Legge GE, Bane MC. Psychophysics of reading. $\mathrm{XV}$ : Font effects in normal and low vision. Invest Ophthalmol Vis Sci. 1996;37( 8):1492-501.

11. Tomioka K. Study on legibility of characters for the elderly - effects of character display modes

12. "CFM reavalia cirurgias de correção visual" In: Portal da Internet Brasil Medicina. Capítulo 49 O Olho I: Óptica da visão. p. 620.[citado 2008 Nov 12]. Disponível em URL: h t t p://www.brasilmedicina.com.br/noticias/ check_printnot.asp?Area $=2 \& \operatorname{Cod}=1321$.

13. Milder B, Rubin ML. Progressive power lenses. Surv Ophthalmol. 1987;32(3):189-98. on legibility. J Physiol Anthropol. 2007;26(2):159-64 .

14. Werner L, Trindade F, Pereira F, Werner L. Fisiologia da acomodação e presbiopia. Arq Bras Oftalmol. 2000;63(6):487-93.

15. Instituto Benjamin Constant. Mitos e verdades sobre oftalmologia. [Internet] [citado 2009 Jan 15]. Disponível em URL: http://www.ibc.gov.br/?itemid=114 .

16. Acesso para todos: ajuda para tornar os processos participativos acessíveis a todos. Save the Children. 2000 [Internet]. [citado 2008 Nov 28]. Disponível em: http:// w w w. e e n e t. org.uk/resources/docs/ access_for_all_portbrazil_final.pdf

17. Instituto Brasileiro de Geografia e Estatística - IBGE. Pesquisa Nacional por amostra de domicílios. 2000 [Internet]. [citado 2008 Nov 28]. Disponível em: http://www.ibge.gov.br/ home/estatistica/populacao/projecao_da_populacao/ publicacao_unfpa.pdf.

\section{Endereço para correspondência:}

José Ricardo C. L. Rehder

Rua Tucumã, no 189 - Apto. 92 - Jardim Europa

CEP 01455-010 - São Paulo (SP), Brasil 